\title{
Worldwide prevalence of fungal coinfections among COVID-19 patients: a comprehensive systematic review and meta-analysis
}

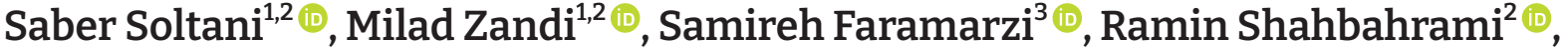 \\ Mohebat Vali $^{4}{ }^{\oplus}$, Sara Akhavan Rezayat ${ }^{5}{ }^{\oplus}$, Reza Pakzad $^{6,7}{ }^{\oplus}$, Pooneh Malekifar $^{8}{ }^{\oplus}$, \\ Iraj Pakzad $^{9}$, ${ }^{\text {Neda Jahandoost }}{ }^{10}{ }^{(1)}$, Jalal Moludi ${ }^{11}$ (1)
}

\author{
${ }^{1}$ Department of Virology, School of Public Health, Tehran University of Medical Sciences, Tehran, Iran \\ ${ }^{2}$ Research Center for Clinical Virology, Tehran University of Medical Sciences, Tehran, Iran \\ ${ }^{3}$ Razi Vaccine and Serum Research Institute, Agricultural Research, Education and Extension Organization (AREEO), Karaj, Iran \\ ${ }^{4}$ Student Research Committee, Shiraz University of Medical Sciences, Shiraz, Iran \\ ${ }^{5}$ Department of Management \& Health Economics, School of Public Health, Tehran University of Medical Sciences, Tehran, Iran \\ ${ }^{6}$ Department of Epidemiology, Faculty of Health, Ilam University of Medical Sciences, Ilam, Iran \\ ${ }^{7}$ Health and Environment Research Center, Ilam University of Medical Sciences, Ilam, Iran \\ ${ }^{8}$ Department of Epidemiology, School of Public Health, Tehran University of Medical Sciences, Tehran, Iran \\ ${ }^{9}$ Department of Microbiology, Faculty of Medicine, Ilam University of Medical Sciences, Ilam, Iran \\ ${ }^{10}$ Department of Microbiology, Faculty of Science, Islamic Azad University, Sanandaj, Iran \\ ${ }^{11}$ School of Nutrition Sciences \& Food Technology, Kermanshah University of Medical Sciences, Kermanshah, Iran
}

Received: November 3, 2021

Revised: December 23, 2021

Accepted: January 2, 2022

Corresponding author:

Reza Pakzad

Department of Epidemiology,

Faculty of Health, Ilam University

of Medical Sciences, Banganjab,

6939177143 llam, Iran

E-mail: rezapakzad2010@yahoo.

com

Co-Corresponding author:

Pooneh Malekifar

Department of Epidemiology,

School of Public Health, Tehran

University of Medical Sciences,

Porsina Street, 14156446

Tehran, Iran

E-mail:malekifar1989@gmail.com

\section{ABSTRACT}

Microbial coinfections can increase the morbidity and mortality rates of viral respiratory diseases. Therefore, this study aimed to determine the pooled prevalence of fungal coinfections in coronavirus disease 2019 (COVID-19) patients. Web of Science, Medline, Scopus, and Embase were searched without language restrictions to identify the related research on COVID-19 patients with fungal coinfections from December 1, 2019, to December 30, 2020. A random-effects model was used for analysis. The sample size included 2,246 patients from 8 studies. The pooled prevalence of fungal coinfections was $12.60 \%$. The frequency of fungal subtype coinfections was 3.71\% for Aspergillus, 2.39\% for Candida, and 0.39\% for other. The World Health Organization's Regional Office for Europe and Regional Office for Southeast Asia had the highest (23.28\%) and lowest (4.53\%) estimated prevalence of fungal coinfection, respectively. Our findings showed a high prevalence of fungal coinfections in COVID-19 cases, which is a likely contributor to mortality in COVID-19 patients. Early identification of fungal pathogens in the laboratory for COVID-19 patients can lead to timely treatment and prevention of further damage by this hidden infection.

Keywords: Coinfection; Coronavirus; COVID-19; Fungi; Meta-analysis

Saber Soltani and Milad Zandi contributed equally to this study as co-first authors. 


\section{Introduction}

Severe acute respiratory syndrome coronavirus 2 (SARSCOV-2), a novel coronavirus, is the causative agent of the recent coronavirus disease 2019 (COVID-19) pandemic [1,2], and it is mainly transmitted by respiratory droplets. The conventional method for diagnosing COVID-19 is a reverse transcription-polymerase chain reaction test [3], but other methods with similar nanotechnology-based strategies can be used for the detection of RNA viruses [4].

Microbial coinfections can increase the mortality rate of COVID-19 patients and lead to prolonged hospitalizations [5]. In particular, fungal coinfections pose a significant threat to COVID-19 patients [6]. Studies have indicated that the detection of fungal coinfections in patients infected with COVID-19 can be difficult and creates a challenging situation when deciding on the proper treatment for these patients; furthermore, fungal coinfections can remain undetectable even after death, thereby skewing estimates of mortality rates [7]. The clinical and radiological features of viral respiratory diseases, such as COVID-19, are similar to those of respiratory diseases caused by fungal pathogens, creating another challenge in diagnosing fungal coinfections [8]. The limited sensitivity of fungal pathogen detection tests is a real problem in identifying fungal pathogens, as exemplified by reports that approximately $50 \%$ of Candida infections are not detectable by blood culture [9]. Various studies have noted that coinfections with fungi such as Aspergillus and Candida can increase mortality rates, especially in critically ill patients [10] such as those hospitalized in critical care wards with COVID-19. Therefore, the prevalence of fungal coinfections in COVID-19 patients should be routinely monitored [10]. In the context of monitoring different types of coinfections among COVID-19 patients, this study aims to identify the prevalence of fungal coinfection. Our goal is to provide evidence that will be valuable for identifying coinfections in COVID-19 patients and help clinicians to provide suitable treatments, especially in the early stages of the disease.

\section{Materials and Methods}

This study was registered in the International Prospective Register of Systemic Reviews (CRD42021240030, https:// www.crd.york.ac.uk/prospero/display_record.php?RecordID =240030) [11]. All required steps of the Preferred Reporting Items for Systematic Reviews and Meta-analysis (PRISMA) protocol were conducted (Suppl. 1). We applied the keywords: COVID-19, coronavirus, SARS-CoV-2 infection, SARS-CoV-2, polymicrobial infection, fungal AND coinfections, fungal
AND secondary infections, and mixed infections for all included studies.

\section{Literature Search}

An in-depth search without language restrictions was performed to find related articles in Web of Science, Medline, Scopus, and Embase. Other databases such as MedRxiv and Social Science Research Network (SSRN) were also reviewed to enhance the accuracy of the search and to obtain any other associated articles. The search period was December 1, 2019, to December 30, 2020. We used Medical Subject Headings (MeSH) keywords for searching the databases. The patient, intervention, comparison, outcome (PICO) framework was applied in this article, as described below for Medline, following the suggestion made by SalvadorOlivan et al. [12]: population/patient: COVID-19 patients; intervention/exposure: none; comparison: none; outcome: fungal coinfection.

The search strategy (Box 1) and keywords used for database searches (Box 2) are available in Suppl. 2.

In addition, we used Google Scholar to explore the gray literature, had a mycologist identify important research, and manually searched reference lists to find related articles. All extracted articles were exported to Endnote ver. X6 and duplicate articles were removed. The screening process was conducted in 3 phases. Articles were first reviewed by title, then by abstract, and finally the full texts were reviewed. To gain access to articles with data that were not publicly available, emails were sent to the corresponding authors. Articles with no response from the corresponding author were excluded. Two independent raters reviewed the 3 phases independently. A third evaluator resolved any inter-rater discrepancies. Study selection processes were conducted based on blinding and task separation. The kappa coefficient for the inter-rater agreement was $94 \%$.

\section{Inclusion and Exclusion Criteria}

Epidemiological research assessing the prevalence of fungal coinfections among COVID-19 cases was recorded without restriction. We excluded case-control studies, clinical trials, and review studies. We also excluded case reports and case series studies with sample sizes less than 10.

\section{Data Extraction}

The author's name, type of study, year of the study, country, sample size, subjects' age and sex, the number of subjects, and fungal species were recorded from the eligible articles. Patients confirmed to have COVID-19 and fungal coinfection were included in the study. 


\section{Variable Definition}

We classified the fungal coinfections based on transmission mode and clinical features. The World Health Organization (WHO) regional classification, which included the Regional Office for Africa, the Regional Office for the Americas, the Regional Office for the Eastern Mediterranean, the Regional Office for Europe, the Regional Office for Southeast Asia, and the Regional Office for the Western Pacific, was used to categorize the countries.

\section{Quality Assessment}

The quality of the studies was evaluated using the NewcastleOttawa Scale [13]. Two researchers independently assessed the articles and, for each study, an overall score was determined. The studies were classified as very good, good, satisfactory, and unsatisfactory [14].

\section{Statistical Analysis}

We performed the statistical analysis with Stata ver. 14.0 (StataCorp., College Station, TX, USA). We also extracted the number of COVID-19 infection cases, the prevalence of COVID-19 with fungal coinfection, and the fungal species. The Cochran $\mathrm{Q}$ test was used to identify heterogeneity as quantified by the $I^{2}$ index, with $I^{2}>0.7$ indicating high heterogeneity. "Metaprop" commands were applied in a forest plot to estimate the pooled prevalence with a $95 \%$ confidence interval (CI). A random-effects model was used to estimate the pooled prevalence [15-20]. Meta-regression analysis was used to evaluate the effect of the WHO region, sample size, and age on heterogeneity. The "metabias" command was used to evaluate publication bias. We adjusted the prevalence rate with the "metatrim" command, using the trim-and-fill method if there was publication bias. Statistical significance was indicated by a $p<0.05$.

\section{Results}

Medline, Scopus, Web of Science, and Embase searches resulted in 1,010 studies, and 41 articles were obtained from other sources. After removing duplicate papers, 510 studies remained. During the first round of screening, 301 studies were excluded, 114 studies were excluded in the second round, and 87 studies were excluded in the third round, leaving 8 studies [21-28] with a sample size of 2,246 cases. Figure 1 shows the selection process flow chart. The different features of the studies are illustrated in Table 1 and more details about coinfections by fungal type are available in Table S1 [21-28]. The European area had the highest number of studies on this subject $(n=4)$ and Southeast Asia provided the lowest number of studies $(n=1)$. All studies were published in 2020. Four case series, 1 cohort study, 1 letter to the editor, and 1 cross-sectional study were included in our research.

\section{Pooled Prevalence of Fungal Coinfections in COVID-19 Patients}

Table 1 shows the prevalence of fungal coinfections in the included studies [21-28]. Figure 2 illustrates the prevalence of fungal coinfections in a forest plot [21-28]. Hughes et al. [24] reported the lowest prevalence of fungal coinfections (prevalence, 0.36\%; 95\% CI, 0.07-1.05) in the United Kingdom and Intra et al. [25], in a study conducted in Italy, reported the highest prevalence of fungal coinfections (prevalence, 31.15\%; 95\% CI, 19.90-44.29). Figure 2 shows the estimated pooled prevalence of fungal coinfections (prevalence, 12.60\%; 95\% CI, 7.84-17.36; $I^{2}, 96.1 \%$; $p<0.001$ ). For every 1,000 people infected with COVID-19, 78 to 173 experienced fungal coinfections.

\section{Pooled Prevalence of Fungal Coinfections Based on Different Subgroups}

Figure 3 provides prevalence data on fungal coinfections according in detail. The most frequent genus among fungal coinfections was Aspergillus (pooled prevalence, 3.71\%; 95\% CI, 0.54-9.11), with other taxa being the least frequent (pooled prevalence, 0.39\%; 95\% CI, 0.01-2.15). The pooled prevalence of Candida was 2.39\% (95\% CI, 0.92-4.42). The highest and lowest pooled prevalence of fungal coinfections occurred in the Regional Office for Europe and Regional Office for Southeast Asia regions, with 23.28\% (95\% CI, 1.6758.41) and 4.53\% (95\% CI, 3.01-6.52), respectively. The pooled prevalence for the Regional Office for the Western Pacific is presented in Figure 3.

\section{Heterogeneity and Meta-Regression}

Significant heterogeneity was found among the studies, as shown in Table $2(p<0.001)$. The $I^{2}$ index for total fungal coinfections and different species was $85 \%$. Meta-regression showed that the WHO region size (coefficient, -0.0118 ; $p=0.924$ ), sample size (coefficient, $-0.001 ; p=0.132$ ), age (coefficient, $-0.0171 ; p=0.110$ ) and quality score (coefficient, 0.001; $p=0.297$ ) had no significant effect on the heterogeneity of the studies (Figure 4).

\section{Publication Bias}

The Egger test showed no significant publication bias in the present meta-analysis. 


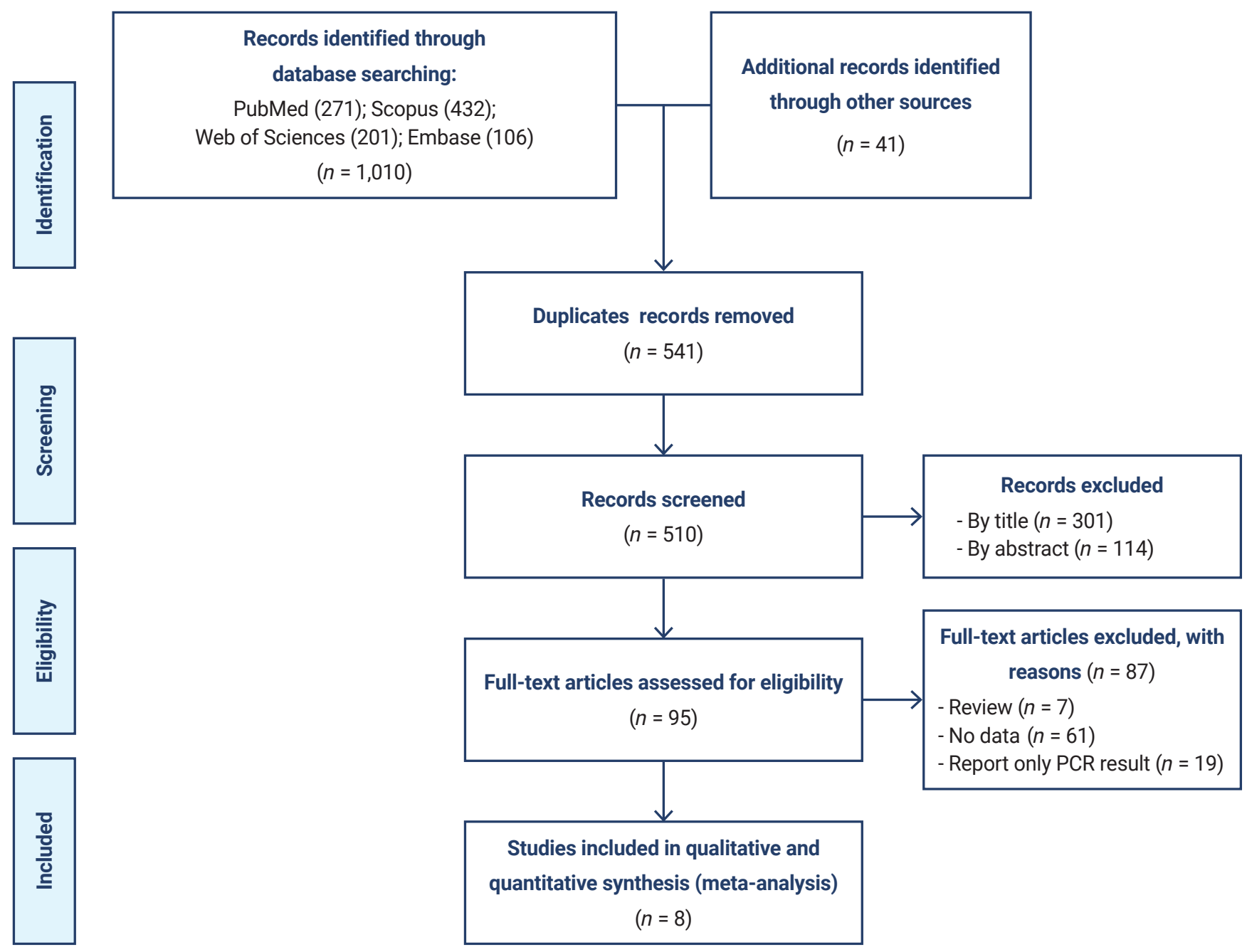

Figure 1. Study flowchart based on the Preferred Reporting Items for Systematic Reviews and Meta-analysis (PRISMA) guideline. PCR, polymerase chain reaction.

Table 1. Characteristics of studies included in the meta-analysis

\begin{tabular}{|c|c|c|c|c|c|c|}
\hline Study & Country & Design & $\begin{array}{c}\text { Publication } \\
\text { year }\end{array}$ & $\begin{array}{c}\text { Mean } \\
\text { age (y) }\end{array}$ & $\begin{array}{l}\text { Sample } \\
\text { size }\end{array}$ & $\begin{array}{l}\text { Fungal coinfection } \\
\text { prevalence }(\%) \\
(95 \% \mathrm{Cl})\end{array}$ \\
\hline Zhu et al. [21] & China & Retrospective case series & 2020 & - & 257 & $23.35(18.31-29.00)$ \\
\hline Agrifoglio et al. [22] & Spain & Letter to the editor & 2020 & 58.7 & 139 & $10.79(6.17-17.17)$ \\
\hline Chowdhary et al. [23] & India & Cross-sectional & 2020 & - & 596 & $2.52(1.41-4.11)$ \\
\hline Hughes et al. [24] & United Kingdom & Retrospective case series & 2020 & 69.5 & 836 & $0.36(0.07-1.05)$ \\
\hline Intra et al. [25] & Italy & Retrospective case series & 2020 & - & 61 & $31.15(19.90-44.29)$ \\
\hline Segrelles-Calvo et al. [26] & Spain & Cohort & 2021 & 59.6 & 215 & $22.79(17.36-28.99)$ \\
\hline Wang et al. [27] & China & Retrospective case series & 2020 & 73 & 104 & $7.69(3.38-14.60)$ \\
\hline Zhang et al. [28] & China & Retrospective cohort & 2020 & 64.76 & 38 & $15.79(6.02-31.25)$ \\
\hline
\end{tabular}

$\mathrm{Cl}$, confidence interval; -, not reported.

\section{Discussion}

The fungal microorganisms in hospitals are neglected factors contributing to the overall health risks of hospitalized patients [29].

Opportunistic infections can often be seen in patients 


\begin{tabular}{|c|c|c|c|}
\hline \multicolumn{2}{|l|}{ First author (year); Country } & \multirow{2}{*}{$\begin{array}{c}\text { Prevalence }(95 \% \mathrm{Cl}) \\
23.35(18.31,29.00)\end{array}$} & \multirow{2}{*}{$\begin{array}{c}\text { Weight (\%) } \\
13.37\end{array}$} \\
\hline Zhu et al. (2020); China & $\longrightarrow$ & & \\
\hline Agrifoglio et al. (2020); Spain & & $10.79(6.17,17.17)$ & 13.24 \\
\hline Chowdhary et al. (2020); India & 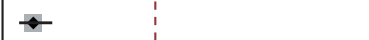 & $2.52(1.41,4.11)$ & 15.88 \\
\hline Hughes et al. (2020); UK & 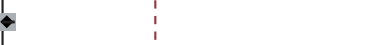 & $0.36(0.07,1.05)$ & 16.05 \\
\hline Intra et al. (2020); Italy & & $31.15(19.90,44.29)$ & 7.82 \\
\hline Segrelles-Calvo et al. (2020); Spain & $\rightarrow$ & $22.79(17.36,28.99)$ & 12.96 \\
\hline Wang et al. (2020); China & $\longrightarrow$ & $7.69(3.38,14.60)$ & 13.14 \\
\hline Zhang et al. (2020); China & & $15.79(6.02,31.25)$ & 7.55 \\
\hline Overall (I-squared $=96.1 \%, p<0.001)$ & & $12.60(7.84,17.36)$ & 100.00 \\
\hline Note: weights are from random effects analysis & & & \\
\hline $\begin{array}{l}1 \\
-10\end{array}$ & 0 & $\begin{array}{l}1 \\
50\end{array}$ & \\
\hline
\end{tabular}

Figure 2. Forest plot of fungal coinfections among coronavirus disease 2019 cases. Each study is denoted in terms of author, year, and region. Each line sector's midpoint shows the estimated prevalence, the length of the line segment shows the $95 \%$ confidence interval $(\mathrm{Cl})$, and the diamond shows the pooled estimate.

infected with respiratory viruses [30]. Microbial agents including Acinetobacter, Klebsiella, Enterobacter, Aspergillus, and Candida are common causative agents of secondary infections in COVID-19 patients [5]. Invasive fungi play an important role among the different types of microbial pathogens causing coinfections in COVID-19 patients and are associated with increased mortality rates [31]. Invasive fungal infections, including Candida and Aspergillus infections, are common among immunocompromised patients in critical condition [32]. Respiratory viruses and fungal pathogens have similar clinical features, which may create difficulties for clinicians in their differential diagnosis, especially at the early onset of the disease [33]. For example, diagnosing a blood infection with Candida is challenging, even today, because of the low number of pathogens in the infected tissue. An estimated 50\% of candidiasis cases cannot be detected by blood culture [34]. This problem is critical because it can interfere with determination of the correct drug to prescribe for patients in the initial stages of the infection [35]. The early detection of COVID-19-associated pulmonary aspergillosis (CAPA) has become problematic. A study in France reported negative serology and culture tests for Aspergillus coinfection in COVID-19 patients during the initial stage of the viral infection, and the CAPA was only confirmed after the patients' deaths [36]. This report shows the importance of early detection of fungal coinfections and the impact of a late diagnosis on increasing the mortality rate. Fungemia has been reported as a complication of SARS-CoV-2 infection [37]. Another risk factor for fungal coinfection could be probiotic consumption, especially in patients admitted to the intensive care unit (ICU). Two patients admitted to the ICU presented with blood infections from Saccharomyces cerevisiae after receiving a probiotic supplement. Data suggested that damage to the intestinal mucosal barrier due to the COVID-19 infection created an opportunity for the fungi to relocate from the probiotics to the bloodstream and cause a blood infection. The study suggests avoiding consumption of prophylactic probiotics by COVID-19 patients in the ICU [38]. Central venous catheterization, frequent use of antibiotics, and steroid therapy are risk factors for fungal coinfection [39]. Different types of antifungal treatment have been used to treat COVID-19 patients infected with fungal pathogens. Antifungal drugs including amphotericin B, micafungin, and fluconazole are some of the main drugs prescribed to treat fungal coinfections [5].

Among Candida species, Candida albicans is the organism most frequently found in critically ill COVID-19 patients. Candida auris is the second most common species causing invasive candidiasis $[39,40]$. Resistance to antifungal therapy is another problem reported in the studies of fungal infection. Multiple studies reported differing percentages of antifungal resistance in $C$. auris. One study indicated that $67 \%$ of the patients who died from an invasive $C$. auris coinfection had received micafungin, yet manifested persistent candidemia [23]. Aspergillus is another frequent 


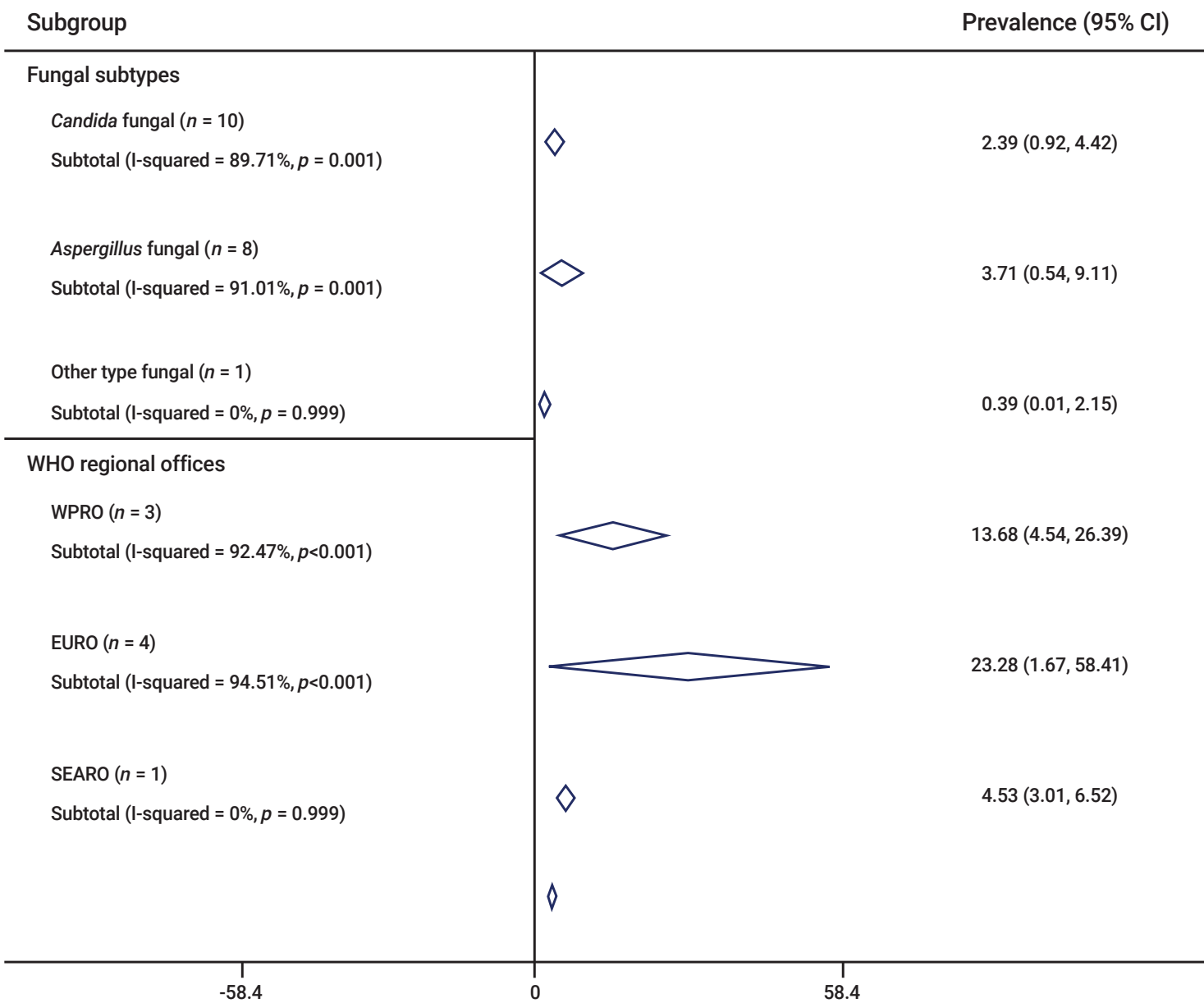

Figure 3. Pooled prevalence with $95 \%$ confidence intervals (Cls) and heterogeneity indices of coronavirus disease 2019 fungal coinfection cases based on fungal taxa and geographic area. The diamond shows the pooled prevalence and the diamond length indicates the $95 \% \mathrm{Cl}$.

WHO, World Health Organization; WPRO, Regional Office for the Western Pacific; EURO, Regional Office for the European Region; SEARO, Regional Office for Southeast Asia.

Table 2. Meta-regression results for the identification of heterogeneity determinants in the studies

\begin{tabular}{lllc}
\hline Variable & Coefficient & \multicolumn{1}{c}{$95 \% \mathrm{Cl}$} & $p$-value \\
\hline Age $(\mathrm{y})$ & -0.0171 & -0.0414 to 0.007 & 0.110 \\
WHO region (score) & -0.0118 & -0.304 to 0.280 & 0.924 \\
Sample size $(n)$ & -0.001 & -0.001 to 0.001 & 0.132 \\
Quality score & 0.001 & -0.002 to 0.002 & 0.297
\end{tabular}

$\mathrm{Cl}$, confidence interval; WHO, World Health Organization.

Coding of WHO regions: Regional Office for the Western Pacific (WPRO), Regional Office for Europe (EURO), Regional Office for Southeast Asia (SEARO).

opportunistic fungal genus. A study conducted in England showed invasive Aspergillus infection to be an underestimated danger in COVID-19 patients. Our study showed that COVID-19 patients had a $12.6 \%$ coinfection rate with Aspergillus [41].
A recent study showed that patients infected with the influenza virus who presented with severe acute respiratory distress syndrome (ARDS), rapidly developed invasive pulmonary aspergillosis (IPA). This finding is concerning because almost $40 \%$ of COVID-19 cases develop ARDS and are therefore susceptible to IPA [42]. A study conducted in China reported a 23.3\% rate of Aspergillus coinfection among COVID-19 patients [21].

Other studies indicated lower rates of Aspergillus coinfection among SARS-CoV-2 cases, ranging from $3.2 \%$ to $5 \%$. It is crucial to note that none of these articles mentioned the specific diagnostic procedures necessary to confirm the presence of fungal pathogens in patients. This may have been because of low sensitivity or the limited availability of gold standard tests during the studies $[42,43]$. CAPA has been widely reported in the European region, up to 35\% [44]. The 

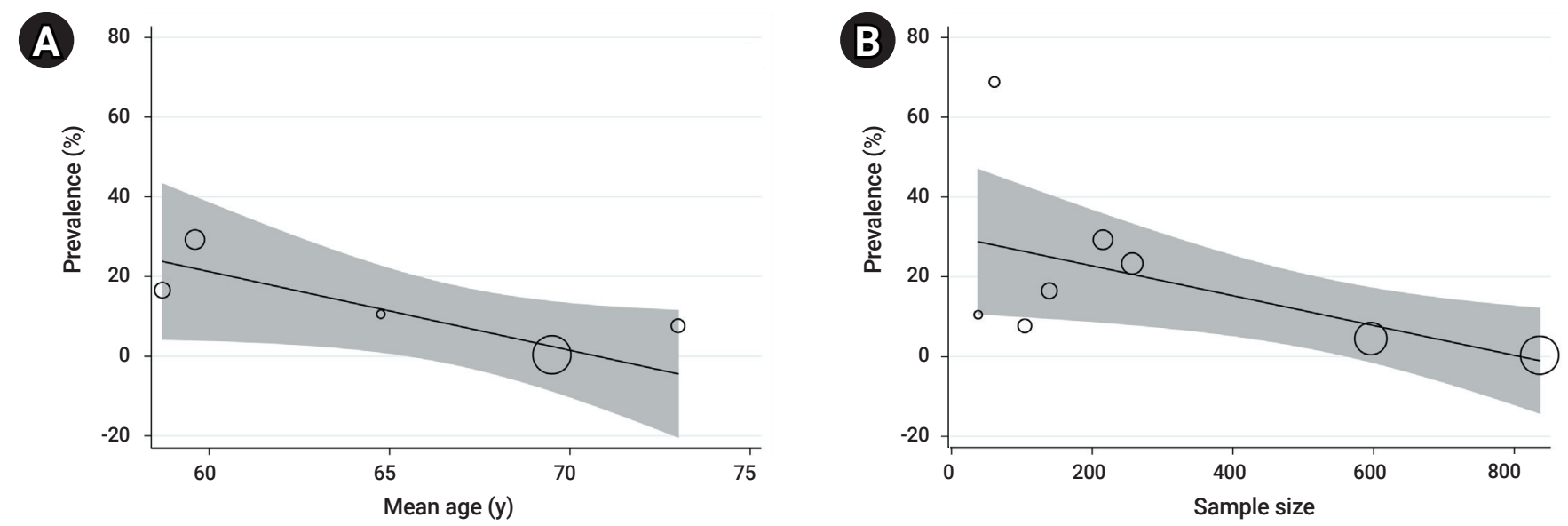

Figure 4. The relationship between age $(A)$ and sample size $(B)$ with the prevalence of fungal coinfections using meta-regression. The circle's size indicates the study's precision. There is no significant association of the prevalence of fungal coinfections with age or sample size.

overall mortality rate among reported case series with confirmed CAPA ranged from $44.5 \%$ to $66.7 \%$ [42]. A metaanalysis performed on fungal coinfection studies reported the overall proportion of fungal coinfections in COVID-19 patients was $0.12 \%$, the overall proportion of Aspergillus was $0.06 \%$, and the overall pooled mortality rate was $0.17 \%$. This study also showed a significant difference when comparing ICU cases with mixed hospitalized populations ( 0.36 vs. 0.06) [45]. Another study reported a $4 \%$ fungal coinfection rate among COVID-19 cases and an $8 \%$ rate of fungal superinfections [46]. Our results showed more consistency, with a uniformly high prevalence of fungal coinfections among COVID-19 patients. We found that Aspergillus was the most frequent fungal taxon in COVID-19 cases and candidiasis the second most frequent fungal coinfection. We also found that the Regional Office for Europe reported the highest coinfection rate $(23.28 \%)$ and that the Regional Office for Southeast Asia reported the lowest coinfection rate $(4.53 \%)$. As previously mentioned, the low rate of reported coinfections in Southeast Asia (especially China) could be because there was a lack of detection equipment or the specific or gold standard tests were not performed to detect fungal coinfections. Our results also support the practical use of antifungal treatment, especially in the early stages of COVID-19 infection, to reduce the mortality rate for critically ill patients. It should be noted that the results of some studies were based on small sample populations and therefore do not provide robust evidence from which to draw conclusions.

\section{Strengths and Limitations}

We faced some limitations while performing our analysis. First, we could not conduct a sex-specific estimation because of limited data in the primary research. Secondly, we estimated the pooled prevalence based on geographical data provided by the WHO. Therefore, our estimations in the spatial analysis of the different geographical areas [4751] were not robust because of inconsistent study numbers. It should be noted that many of the studies suffered from significant sources of bias, but we assessed the quality of the included studies and considered the effect of the quality score as a source of heterogeneity. The meta-regression analysis showed that the quality score did not affect the pooled frequency estimates. Finally, in many instances, the analysis was based on very few studies. Therefore, the evidence supporting the results is low, and the findings must be interpreted with caution. Nonetheless, strength of this study is that it performed a comprehensive search and meta-analysis to determine the pooled prevalence of different fungal subtypes. Another limitation in our study is the significant heterogeneity and difficulty in interpreting results due to different sets of data, including various regimens, drug doses, durations, center settings, and population samples. The fact that many of the reviewed studies suffered from significant sources of bias should also be taken into consideration.

\section{Conclusion}

Our results showed that $12.60 \%$ of COVID-19 patients were infected with fungal pathogens. We also found that Aspergillus and Candida were the most frequent fungal genera among the patients. Due to the difficulties in detecting fungal coinfections, particularly in the initial stages of COVID-19 infection, we support the routine use of antifungal treatments in COVID-19 patients. 


\section{Supplementary Material}

Table S1. Details of fungal coinfections in included studies; Suppl. 1. PRISMA 2020 checklist; Suppl. 2. Search strategy for Medline (MeSH, Medical Subject Headings). Supplementary data are available at https://doi.org/10.24171/j.phrp.2021.0293.

\section{Notes}

\section{Ethics Approval}

Not applicable.

\section{Conflicts of Interest}

The authors have no conflicts of interest to declare.

\section{Funding}

None.

\section{Availability of Data}

All data extracted and analyzed during this study are included in this published article. For other data, these may be available through the corresponding author upon reasonable request.

\section{Authors' Contributions}

Study Design and Idea: SS; Conceptualization: JM, NJ, MZ, RS; Data curation: SAR; MZ, SF; Formal analysis: RP, PM, RS; Funding acquisition: all authors; Investigation: IP, SAR, SF; Methodology: RP, PM; Project administration: RS, SS, RP; Resources: JM, NJ, SF; Software: RP, PM; Supervision: IP, MV, SS, RP; Validation: JM, NJ, MV; Visualization: MV; Writing-original draft: all authors; Writing-review $\&$ editing: all authors.

\section{Additional Contributions}

I would like to express our very great appreciation to Ilam University of Medical Sciences, Ilam and Tehran University of Medical Sciences, Iran.

\section{References}

1. Zandi M, Soltani S. Hemagglutinin-esterase cannot be considered as a candidate for designing drug against COVID-19. Mol Divers 2021;25:1999-2000.

2. Soltani $S$. The hemagglutinin-esterase gene in human coronaviruses SARS-CoV-2, HKU1 and OC43. Eur Rev Med Pharmacol Sci 2020;24: 6484-5.

3. Fani M, Zandi M, Soltani S, et al. Future developments in biosensors for field-ready SARS-CoV-2 virus diagnostics. Biotechnol Appl Biochem 2021;68:695-9.

4. Mozhgani SH, Kermani HA, Norouzi M, et al. Nanotechnology based strategies for HIV-1 and HTLV-1 retroviruses gene detection. Heliyon 2020;6:e04048.

5. Silva DL, Lima CM, Magalhaes VCR, et al. Fungal and bacterial coinfections increase mortality of severely ill COVID-19 patients. J Hosp Infect 2021;113:145-54.

6. Vincent JL, Rello J, Marshall J, et al. International study of the prevalence and outcomes of infection in intensive care units. JAMA 2009;302:2323-9.

7. Gangneux JP, Bougnoux ME, Dannaoui E, et al. Invasive fungal diseases during COVID-19: we should be prepared. J Mycol Med 2020;30:100971.

8. Feldman C, Anderson R. The role of co-infections and secondary infections in patients with COVID-19. Pneumonia (Nathan) 2021;13:5.

9. Clancy CJ, Nguyen MH. Finding the "missing 50\%" of invasive candidiasis: how nonculture diagnostics will improve understanding of disease spectrum and transform patient care. Clin Infect Dis 2013;56:1284-92.

10. Song G, Liang G, Liu W. Fungal co-infections associated with global COVID-19 pandemic: a clinical and diagnostic perspective from China. Mycopathologia 2020;185:599-606.

11. Moher D, Liberati A, Tetzlaff J, et al. Preferred reporting items for systematic reviews and meta-analyses: the PRISMA statement. PLoS Med 2009;6:e1000097.

12. Salvador-Olivan JA, Marco-Cuenca G, Arquero-Aviles R. Errors in search strategies used in systematic reviews and their effects on information retrieval. J Med Libr Assoc 2019;107:210-21.

13. Wells GA, Shea B, O'Connell D, et al. The Newcastle-Ottawa Scale (NOS) for assessing the quality of nonrandomised studies in metaanalyses [Internet]. Ottawa: Ottawa Hospital Research Institute; [cited 2022 Jan 8]. Available from: http://www.ohri.ca/programs/ clinical_epidemiology/oxford.asp.

14. Hashemi H, Pakzad R, Yekta A, et al. Global and regional prevalence of age-related cataract: a comprehensive systematic review and meta-analysis. Eye (Lond) 2020;34:1357-70.

15. Hallajzadeh J, Khoramdad M, Izadi N, et al. The association between metabolic syndrome and its components with systemic lupus erythematosus: a comprehensive systematic review and metaanalysis of observational studies. Lupus 2018;27:899-912.

16. Soltani S, Faramarzi S, Zandi M, et al. Bacterial coinfection among coronavirus disease 2019 patient groups: an updated systematic review and meta-analysis. New Microbes New Infect 2021;43:100910.

17. Sharifi N, Dolatian M, Fathnezhad A, et al. Prevalence of low birth weight in Iranian newborns: a systematic review and meta-analysis. Int J Women's Health Reprod Sci 2018;6:233-9.

18. Mohammadzadeh F, Dolatian M, Jorjani M, et al. Urogenital chlamydia trachomatis treatment failure with azithromycin: a meta-analysis. Int J Reprod Biomed 2019;17:603-20.

19. Soltani S, Tabibzadeh A, Zakeri A, et al. COVID-19 associated central nervous system manifestations, mental and neurological symptoms: a systematic review and meta-analysis. Rev Neurosci 2021;32:351-61.

20. Behesht Aeen F, Pakzad R, Goudarzi Rad M, et al. Effect of prone position on respiratory parameters, intubation and death rate in COVID-19 patients: systematic review and meta-analysis. Sci Rep 2021;11:14407.

21. Zhu X, Ge Y, Wu T, et al. Co-infection with respiratory pathogens among COVID-2019 cases. Virus Res 2020;285:198005.

22. Agrifoglio A, Cachafeiro L, Figueira JC, et al. Critically ill patients with COVID-19 and candidaemia: We must keep this in mind. J Mycol Med 2020;30:101012. 
23. Chowdhary A, Tarai B, Singh A, et al. Multidrug-resistant Candida auris infections in critically Ill coronavirus disease patients, India, April-July 2020. Emerg Infect Dis 2020;26:2694-6.

24. Hughes S, Troise O, Donaldson H, et al. Bacterial and fungal coinfection among hospitalized patients with COVID-19: a retrospective cohort study in a UK secondary-care setting. Clin Microbiol Infect 2020;26:1395-9.

25. Intra J, Sarto C, Beck E, et al. Bacterial and fungal colonization of the respiratory tract in COVID-19 patients should not be neglected. Am J Infect Control 2020;48:1130-1.

26. Segrelles-Calvo G, Araujo GRS, Llopis-Pastor E, et al. Prevalence of opportunistic invasive aspergillosis in COVID-19 patients with severe pneumonia. Mycoses 2021;64:144-51.

27. Wang J, Yang Q Zhang P, et al. Clinical characteristics of invasive pulmonary aspergillosis in patients with COVID-19 in Zhejiang, China: a retrospective case series. Crit Care 2020;24:299.

28. Zhang H, Zhang Y, Wu J, et al. Risks and features of secondary infections in severe and critical ill COVID-19 patients. Emerg Microbes Infect 2020;9:1958-64.

29. Mohajeri P, Soltani S, Getso MI, et al. Investigation of bio-air contamination in some hospitals of Kermanshah, Iran. Adv Hum Biol 2019;9:65-70.

30. Sharifipour E, Shams S, Esmkhani M, et al. Evaluation of bacterial co-infections of the respiratory tract in COVID-19 patients admitted to ICU. BMC Infect Dis 2020;20:646.

31. Hoenigl M. Invasive fungal disease complicating coronavirus disease 2019: when it rains, it spores. Clin Infect Dis 2021;73:e1645-8.

32. Low $\mathrm{CY}$, Rotstein $\mathrm{C}$. Emerging fungal infections in immunocompromised patients. F1000 Med Rep 2011;3:14.

33. Chibabhai V, Duse AG, Perovic O, et al. Collateral damage of the COVID-19 pandemic: exacerbation of antimicrobial resistance and disruptions to antimicrobial stewardship programmes? S Afr Med J 2020;110:572-3.

34. Clancy CJ, Nguyen MH. Diagnosing invasive candidiasis. J Clin Microbiol 2018;56:e01909-17.

35. de Pauw BE. Fungal infections: diagnostic problems and choice of therapy. Leuk Suppl 2012;1(Suppl 2):S22-3.

36. Blaize M, Mayaux J, Nabet C, et al. Fatal invasive aspergillosis and coronavirus disease in an immunocompetent patient. Emerg Infect Dis 2020;26:1636-7.

37. Bishburg E, Okoh A, Nagarakanti SR, et al. Fungemia in COVID-19 ICU patients, a single medical center experience. J Med Virol 2021; 93:2810-4.
38. Ventoulis I, Sarmourli T, Amoiridou P, et al. Bloodstream infection by Saccharomyces cerevisiae in two COVID-19 patients after receiving supplementation of Saccharomyces in the ICU. J Fungi (Basel) 2020; 6:98.

39. Arastehfar A, Carvalho A, Nguyen MH, et al. COVID-19-associated candidiasis (CAC): an underestimated complication in the absence of immunological predispositions? J Fungi (Basel) 2020;6:211.

40. Rodriguez JY, Le Pape P, Lopez O, et al. Candida auris: a latent threat to critically Ill patients with coronavirus disease 2019. Clin Infect Dis 2021;73:e2836-7.

41. White PL, Dhillon R, Cordey A, et al. A national strategy to diagnose coronavirus disease 2019-associated invasive fungal disease in the intensive care unit. Clin Infect Dis 2021;73:e1634-44.

42. Arastehfar A, Carvalho A, van de Veerdonk FL, et al. COVID-19 associated pulmonary aspergillosis (CAPA)-from immunology to treatment. J Fungi (Basel) 2020;6:91.

43. Chindamporn A, Chakrabarti A, Li R, et al. Survey of laboratory practices for diagnosis of fungal infection in seven Asian countries: an Asia Fungal Working Group (AFWG) initiative. Med Mycol 2018;56:416-25.

44. Rutsaert L, Steinfort N, Van Hunsel T, et al. COVID-19-associated invasive pulmonary aspergillosis. Ann Intensive Care 2020;10:71.

45. Peng J, Wang Q Mei H, et al. Fungal co-infection in COVID-19 patients: evidence from a systematic review and meta-analysis. Aging (Albany NY) 2021;13:7745-57.

46. Musuuza JS, Watson L, Parmasad V, et al. Prevalence and outcomes of co-infection and superinfection with SARS-CoV-2 and other pathogens: a systematic review and meta-analysis. PLoS One 2021;16:e0251170.

47. Mahdavifar N, Pakzad R, Ghoncheh M, et al. Spatial analysis of breast cancer incidence in Iran. Asian Pac J Cancer Prev 2016;17(S3):59-64.

48. Pakzad R, Moudi A, Pournamdar Z, et al. Spatial analysis of colorectal cancer in Iran. Asian Pac J Cancer Prev 2016;17(S3):53-8.

49. Pakzad R, Ghoncheh M, Pournamdar Z, et al. Spatial analysis of skin cancer incidence in Iran. Asian Pac J Cancer Prev 2016;17(S3):33-7.

50. Pakzad R, Khani Y, Pakzad I, et al. Spatial analysis of stomach cancer incidence in Iran. Asian Pac J Cancer Prev 2016;17(S3):27-32.

51. Pakzad R, Pakzad I, Safiri S, et al. Spatiotemporal analysis of brucellosis incidence in Iran from 2011 to 2014 using GIS. Int J Infect Dis 2018;67: 129-36. 